\title{
Blocking Artifacts Reduction in Block based Discrete Cosine Transform Compressed Images
}

\author{
Parmjeet Kaur \\ Assistant Professor, \\ Department of Electronics and \\ Communication Engineering, \\ College of Engineering and \\ Management, \\ Kapurthala.
}

\author{
Poonam Sethi \\ Assistant Professor, \\ Department of Electronics and \\ Communication Engineering, \\ DAV Institute of Engineering \\ and Technology,
}

Jalandhar

\begin{abstract}
Image compression is a technique to reduce the volume of information to be transmitted. Compression technique reduces the bandwidth and the volume of the information to be transmitted. We can compress audio signal, video signal, text, fax and images. For medical images lossless compression is used and for other types lossy compression can be used. For compressing an image we can use the DCT technique. But after compression during decompression and recovering original image from compressed image we can face problem of blocking artifacts. Various methods can be used for removing blocking artifacts. One of them is DCT filtering. Further for getting better results we can remove blocking artifacts by spatial and hybrid filtering method. Our experimental results shows that hybrid filtering gives better performance on the bases of better PSNR, BER and MSE.
\end{abstract}

\section{Keywords}

DCT, Spatial filtering, Hybrid filtering, JPEG, Blocking artifacts, image compression, MSE, BER, PSNR.

\section{INTRODUCTION}

Image processing is the method to improve the original images obtained from camera or any other source. Many image processing techniques can be used. One of the processing techniques, image compression is used to reduce the amount of data required to represent an image. Compressing an image is significantly different than compressing raw binary data. This is because images have certain statistical properties which can be exploited by encoders specifically designed for them. Also, some of the finer details in the image can be ignored for the sake of saving a little more bandwidth or storage space. The objective of image compression is to reduce irrelevance and redundancy of the image data in order to be able to store or transmit (text, fax, images) data in an efficient form. Now a day's Transform coding is the most commonly used method for this. In transform coding, Fourier related transform such as DCT or wavelet transform are applied, followed by quantization and entropy coding. Compression artifacts occur in many common media such as DVD's, common computer file formats such as JPEG, MP3 and MPEG files. Image compression may be lossy or lossless [1]. Lossless compression is preferred for archival purposes and often for medical imaging. Lossy compression methods, especially when used at low bitrates, introduce compression artifacts and are especially suitable for natural images such as photographs in applications where minor loss of fidelity is acceptable to achieve a substantial reduction in bit rate. With the development of signal processing, compression technology has been widely applied in various applications, including digital cameras, DVD and broadcast. The process of image compression is required in many imaging and video applications. However, these applications are all bothered by an annoying problem i.e. blocking artifacts.

\section{BLOCKING ARTIFACTS}

Blocking artifacts are the major coding artifacts caused by high compression. These appear as grid noise along the block boundaries in smooth areas and are caused due to independent encoding of each block without considering the correlation between adjacent blocks. To reduce irrelevancy, redundancy of the image data and volume of data to be transmitted, image compression is must. However with the use of lossy image compression methods blocking artifacts get introduced. A compression artifact is a noticeable distortion of media which may be an image, audio or video, due to the application of an overly aggressive or inappropriate lossy data compression algorithm. These lossy data compression schemes discard some data to simplify the media sufficiently to store it in the desired space (data-rate). If there is not enough data in the compressed version to reproduce the original with acceptable fidelity, artifacts will result. Alternatively, the compression algorithm may incorrectly determine certain distortions to be of little subjective importance but they may in fact be objectionable to the viewer. The artifacts such as over smoothing of images, degradation due to quantization errors, ringing effects, blurring of images, blocking noise, image features irregularities etc. So to overcome these artifacts, various methods are used such as Normal DCT filtering, Spatial Filtering, Hybrid Filtering methods, DWT, Adaptive Fuzzy Filtering method, iterative reconstruction algorithm etc. The boundary regions between blocks are identified as either smooth or non-smooth regions [2]. The blocking artifacts in smooth regions are removed by modifying a few DCT coefficients appropriately, whilst an edge-preserving smoothing filter is applied to the non-smooth regions. Above mentioned algorithms are highly efficient in removing blocking artifacts and produce visuality that is much more pleasing to the eye. 


\section{METHODS FOR REMOVAL OF BLOCKINGARTIFACTS}

The DCT is used for two international image and video compression standards, Joint Photographic Experts Group (JPEG) and Motion Picture Experts Group (MPEG).This method are computationally faster and can efficiently eliminate blocking effects for images coded at different bit rates without producing noticeable artifacts. It smoothes out the undesired block edges while retaining the sharpness of the decoded image to a noticeable degree. Its algorithm requires a fairly low computational effort [9-10]. Thus it is useful for real-time applications.

\subsection{Discrete Cosine Transform}

DCT is a Fourier-related transform similar to the discrete Fourier transform (DFT), but using only real numbers. DCTs are equivalent to DFTs of roughly twice the length, operating on real data with even symmetry, while in some variants the input and output data are shifted by half a sample. The DCT is used in JPEG image compression, and MPEG. There, the twodimensional DCT-II of [ $\mathbf{N} \times \mathbf{N}]$ blocks is computed and the results are quantized and entropy coded. In this case, $N$ is typically 8 and the DCT-II formula is applied to each row and column of the block. The result is an $[8 \times 8]$ transform coefficient array.DCT is very efficient method used in image compression and processing of images .It is easy to implement this method in MATLAB. Its mathematical equation is easy to implement through MATLAB programming.

\subsection{Spatial filtering}

Spatial filtering is a term used to describe the methods used to compute spatial density estimates for events observed at individual locations. Spatial prediction does not out-perform pure DCT based technique (such as JPEG) in terms of bit-rate tradeoff. However, at very low bit rates it results in far fewer blocky artifacts and markedly better visual quality. It describes a set of tools for displaying functions estimated from these data points that are distributed in two-dimensional space. The algorithm employs a two-dimensional (2-D) filter in the areas away from edges, and for near edges, onedimensional (1-D) filter aligned parallel to edge so as to reduce the blocking artifacts. So for removing blocking artifacts near edges we need spatial filtering.

\subsection{Hybrid filtering}

Hybrid filtering method is used to combine both DCT and spatial filtering methods discussed already in previous section. There is no need of IDCT in hybrid filtering method. So hardware implementation of this method is easy. Ringing effect can be reduced by hybrid filtering method. This method can give better performance in terms of both objective and subjective views than the previous methods. This method can be seen as a hybrid approach, because it is applied to the blocks with vertical or horizontal edge [14]. The hybrid method works by combining the filtering methods for three different grayscale gradient ranges respectively. It is used to get better processing speed, reduce blurring and enhance edges to a large extent. Therefore it can be used for highspeed noise elimination of 3D images with large data. We can implement this method by MATLAB.

\subsection{Application of DCT in Loss Image Compression}

The DCT transform coding method compresses image data by representing the original signal with a small number of transform coefficients. It exploits the fact that for typical images a large amount of signal energy is concentrated in a small number of coefficients. The goal of DCT transform coding is to minimize the number of retained transform coefficients while keeping distortion at an acceptable level. This DCT is done in $8 \times 8$ non overlapping blocks. Block transform coding divides an image into blocks of equal size and processes each block independently. Block processing allows the coder to adapt to local image statistics, exploit the correlation present among neighboring image pixels and to reduce computational and storage requirements. Larger blocks do not offer significantly better compression, since correlation between pixels tends to decrease as the block size grows. To get best image quality at a given bit- rate (or compression rate) is the main goal and this can be achieved by using different DCT functions for image compression and for different blocking artifacts reduction methods.

\section{LITERATURE STUDY}

There have numerous numbers of researches on image compression and blocking artifacts reductions. There are number of filtering techniques. An advanced discrete cosine transform (DCT)-based image compression method that combined advantages of several approaches. Image compression using Discrete Cosine Transform (DCT) is one of the simplest commonly used compression methods. The quality of compressed images is marginally reduced at higher compression ratios due to the lossy nature of DCT compression and an optimum DCT compression ratio. And an ideal image compression system that yield high quality compressed images with good compression ratio, while maintaining minimum time cost. Firstly divide an image into blocks of different sizes by a rate-distortion-based modified horizontal-vertical partition scheme. Statistical redundancy of quantized DCT coefficients of each image block is reduced by a bit-plane dynamical arithmetical coding with a sophisticated context modeling. This post-filtering removes blocking artifacts in decompressed images. This method provides significantly better compression than JPEG and other DCTbased techniques. Image compression is a very important issue for several applications in the area of multimedia communications, the objective being reduction of storage and transmission costs. However, the reconstructed images from JPEG compression produce annoying blocking artifacts near block boundaries, particularly in highly compressed images. The blocking artifacts in smooth regions were removed by modifying a few DCT coefficients appropriately, whilst an edge-preserving smoothing filter was applied to the nonsmooth regions. Block edge artifacts is one of the most noticeable impairment associated with MPEG and other block based encoding techniques. Blocking artifacts can be removed using variable width non-linear filtering as well as randomized masking of geometric patterns associated with blockiness. High-frequency details of the coded images are mainly contaminated by quantization noise. Non-linear adaptive filter to reduce both staircase and grid noises in decompressed images without any additional information and without introducing significant blurring. The reconstructed images from JPEG compression produced noticeable image degradation near the block boundaries, in particular for highly compressed images, because each block was transformed and 
quantized independently. It was an adaptive approach which performed blockiness reduction in both the DCT and spatial domains to reduce the block-to-block discontinuities. This post- processing algorithm, which consisted of three stages, reduced these blocking artifacts efficiently.

\section{PROBLEM FORMULATION}

The image compression is used to minimize the number of bits needed to represent an image without sacrificing quality of image. However, when we compress the information, then we face the problem of unwanted blocking artifacts. Blocking artifacts can take on several forms in DCT compressed images. These artifacts are visually very annoying and have a substantial impaction to the subjectively perceived image quality. The artifacts removal algorithms are cost effective and yield better performance in terms of both objective and subjectively.

\subsection{Blocking Artifacts reduction algorithms}

Blocking Artifacts reduction algorithms give significant improvement in the separation between blocking noise, image features and effective reduction of image blurring. Many steps are to be performed to preserve the global and local edges of the processed image, remove blocking noise and ensure smoothness without blurring. It helps to remove blocking artifacts and to enhance feature regularities [18]. In general the DCT based method required as much as six times more processing time as compared to the spatial method. The time in DCT methods will be always more as a lot of processing is involved in conversion to and from the DCT coefficients. Spatial filtering is an effective method for improving JPEG, MPEG compression in fixed and variable rate control applications. The method Hybrid Filtering yields better performance in terms of both objective and subjective views than the conventional methods. Hybrid filtering method is nonlinear, computationally efficient, and spatially adaptive.

\subsection{Steps for the Research Work}

Step1. To compress image using DCT technique.

Step2.To remove blocking artifacts using DCT filtering, spatial filtering and hybrid filtering method.

Step3.To compares the above techniques qualitatively and quantitatively on the basis of parameters such as PSNR, MSE and BER.

Table 1. Various Parameters

\begin{tabular}{|l|c|c|c|}
\hline $\begin{array}{l}\text { Filtering } \\
\text { Technique. }\end{array}$ & MSE & PSNR & BER \\
\hline $\begin{array}{l}\text { DCT } \\
\text { filtering }\end{array}$ & 30.5695 & 33.2779 & 0.0300 \\
\hline $\begin{array}{l}\text { Spatial } \\
\text { Filtering }\end{array}$ & 11.4668 & 37.5364 & 0.0266 \\
\hline $\begin{array}{l}\text { Hybrid } \\
\text { filtering }\end{array}$ & 0.8204 & 48.9905 & 0.0204 \\
\hline
\end{tabular}

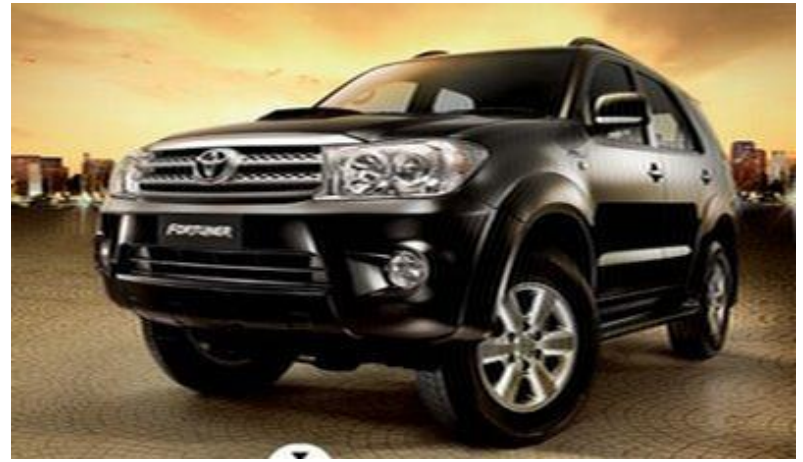

Fig 1: Original image before compressed.
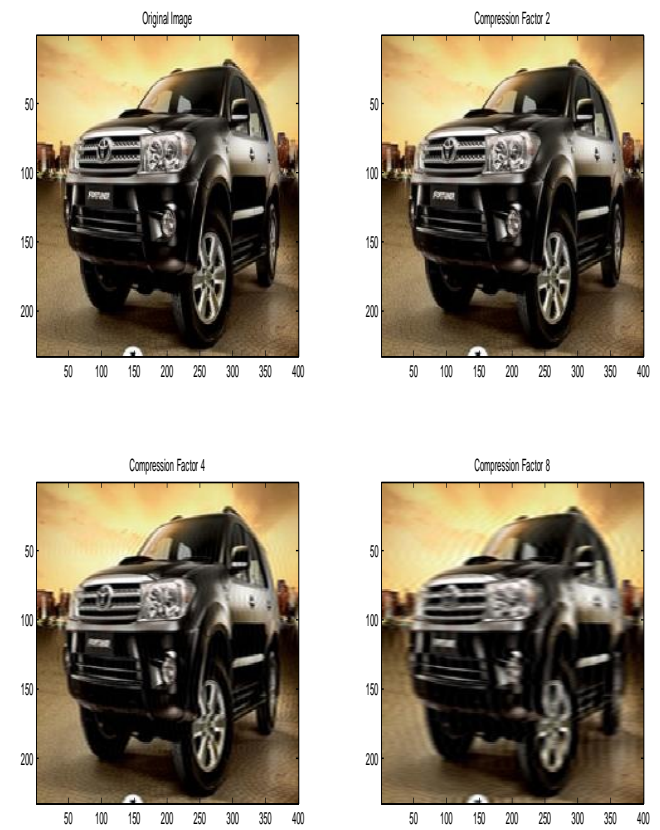

Fig 2: Images obtained after DCT compression, with compression factor $2,4, \& 8$ respectively.

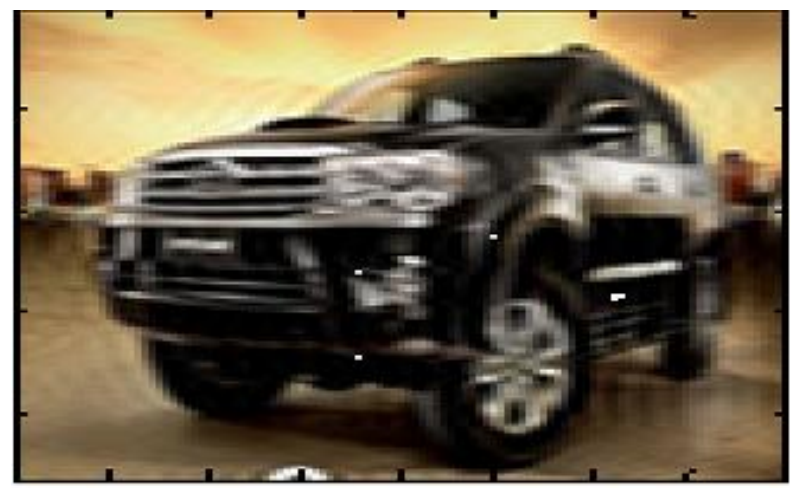

Fig 3: Image having Blocking Artifacts. 
Table 2. Table showing Images recovered after Filtering by three different methods and their graphs of MSE, BER\&PSNR.

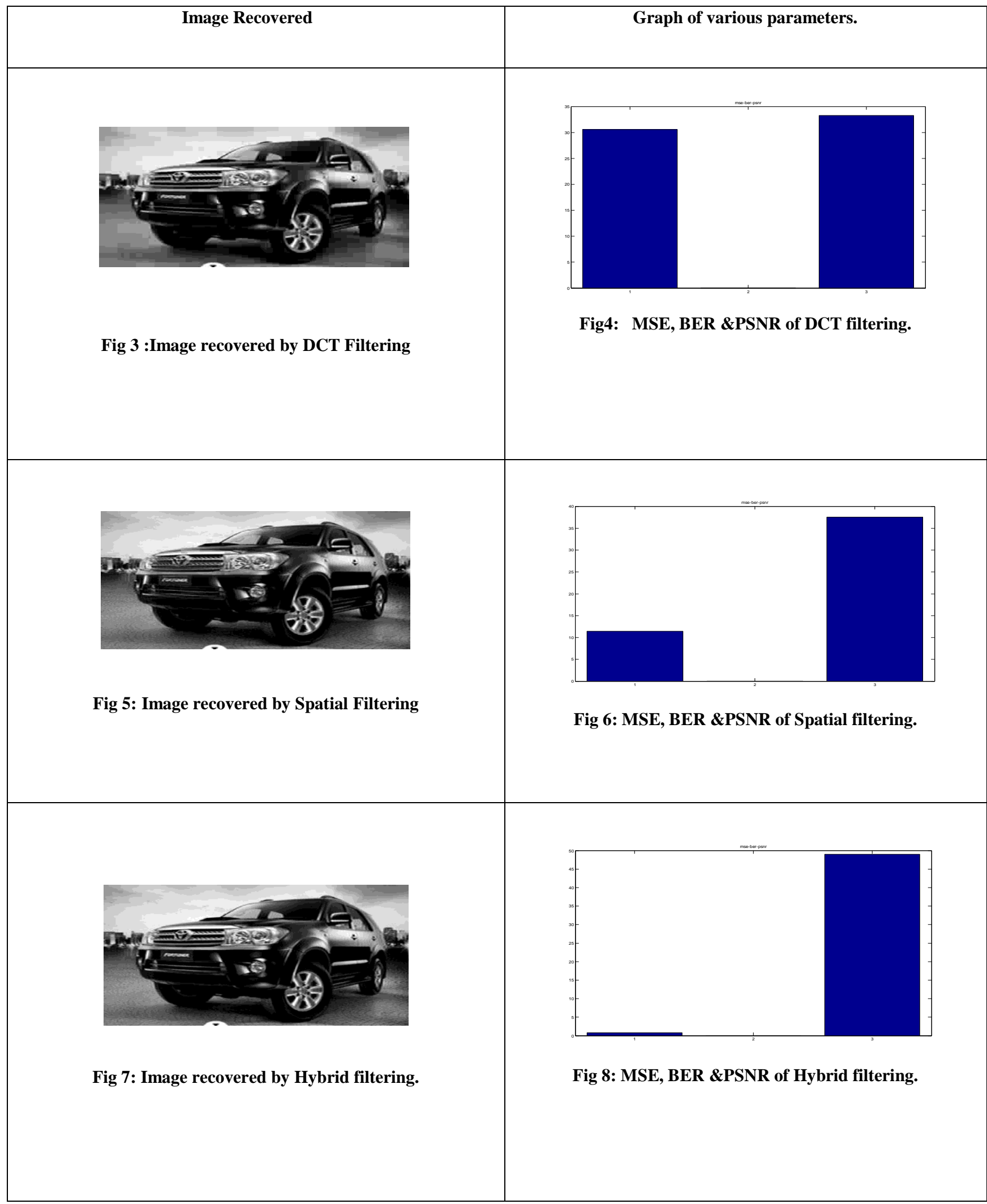


Table 3. Graphs showing comparison of various methods of Removing Blocking Artifacts.

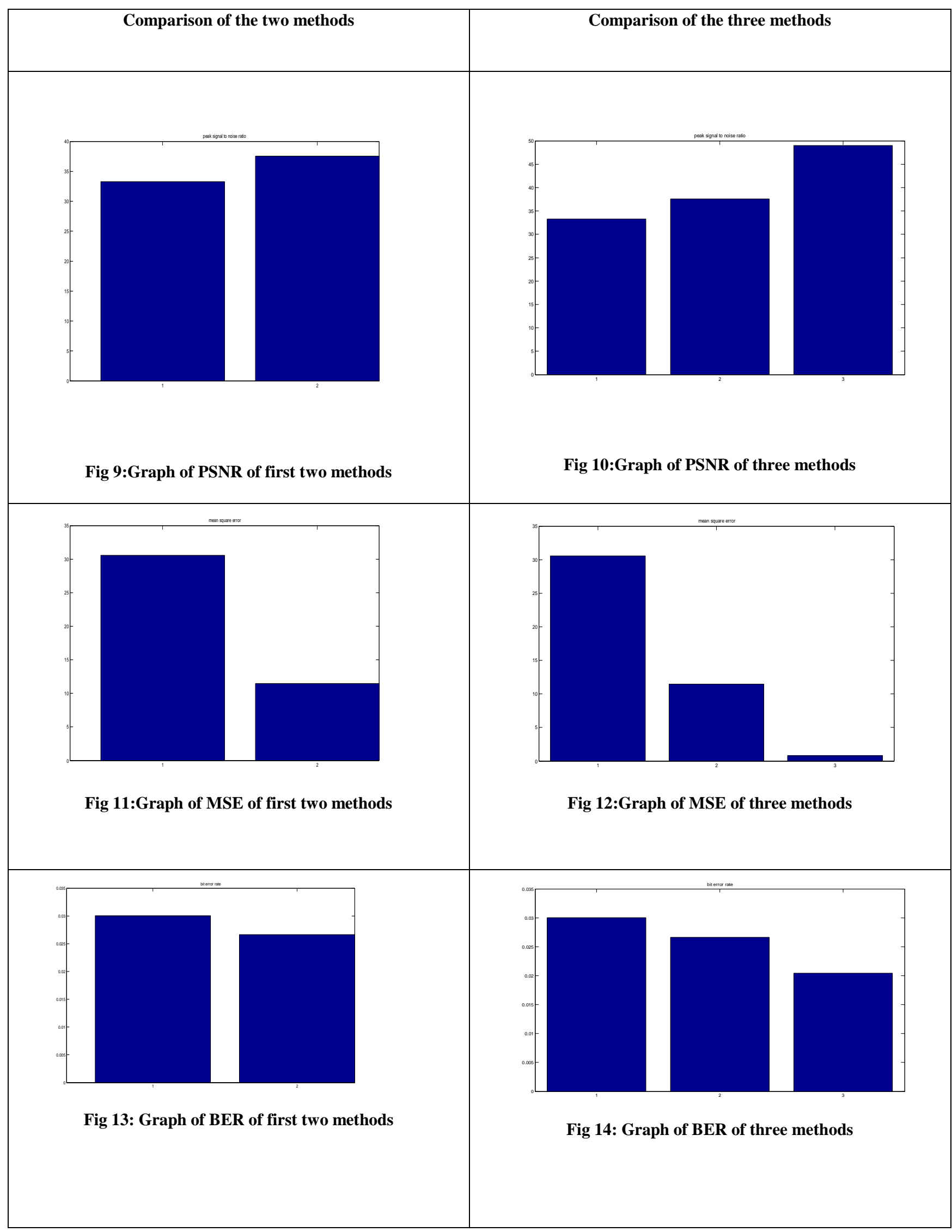




\section{EXPERIMENTAL RESULTS}

By using MATLAB R2010a version 7.10.0 software we observed the results, that are shown in Table1, Table2 and Table3 respectively. Firstly we take the original image then compress it using DCT compression we concluded that as we increase the compression factor the blurring of the image increases. After that when image is decompressed for recovering the original image, it shows blocking artifacts in it. To remove those blocking artifacts we need to use the filtering techniques. So we use the filtering technique DCT, Spatial and hybrid.Table1, shows the various parameters like BER (Bit Error Rate), MSE (Mean Square Error), PSNR (Peak Signal to Noise Ratio) of these three techniques.Table2, shows the output of three techniques and graphical representation of various parameters.Table3, shows the graphical representation of the comparison of the BER, PSNR, MSE .Where PSNR is peak signal to noise ratio in decibels, between the two images. This ratio is often used as a quality measurement between the original image and the compressed image. The higher the PSNR, the better the quality of the compressed or reconstructed image. MSE\& PSNR are the two error matrices used to compare image compression quality. The MSE represents the cumulative squared error between the compressed and original image, whereas PSNR represents the measure of the peak error. The lower the value of MSE the lower the error.BER is bit error rate and we determine how many of the received bits are in error, and then compute the bit-error-rate as the number of bit errors divided by the total number of bits in the transmitted signal.

\subsection{CONCLUSION}

We concluded that both qualitatively and quantitatively the spatial filtering technique is better than the DCT filtering and hybrid filtering is better than the spatial filtering. Because when we compare first two techniques then we observed that PSNR of second method is more than first and MSE and BER are less. And when we compare the three methods than we observed that hybrid is having good PSNR and lesser value of BER and MSE than the first two methods. As we know that for better filtering and recovering of images we need more PSNR and less BER and MSE so hybrid filtering is better than the both methods.

\section{REFERENCES}

[1] Nikolay N. Ponomarenko, Karen O. Egiazarian, Vladimir V.Lukin, "High-Quality DCT-Based Image Compression Using Partition Schemes", IEEE Signal Processing Letters, Vol. 14, No. 2, pp.105-108, Feb. 2007.

[2] Sukhwinder Singh, Vinod Kumar, H.K. Verma, "Reduction of blocking artifacts in JPEG compressed images", Digital Signal Processing, Vol. 17, pp. 225243, Jan. 2007.

[3] Ekaterina BarzykinaRabab K. Ward ,"Removal of Blocking Artifacts using Random Filtering", IEEEimage processing, Vol. 2, pp. 904-908, oct.1999.

[4] Amjed S. Al-Fahoum and Ali M. Reza, "Combined Edge Crispiness and Statistical Differencing for Deblocking
JPEG Compressed Images", IEEE Transactions on Image Processing, Vol. 10, No. 9, pp. 1057-7149, Sep. 2001.

[5] Massinio Mancuso, Monica Besana, RinaldoPoluzzi, “A new post processing algorithm To reduce block artifacts in block coded images", IEEE Microelectronics, Vol. 43, pp.303-307, Aug. 1997.

[6] Shizhong Liu, Student Member, and Alan C. Bovik, Fellow,"Efficient DCT-Domain Blind Measurement and Reduction of Blocking Artifacts", IEEE Circuits \& Systems for video Technology, Vol.12, No.12, pp.11391149 , Dec. 2002

[7] F. M. Bayer and R. J. Cintra Member, "Image Compression via a Fast DCT Approximation", IEEE Latin America Transactions, Vol. 8, No. 6, pp.708-713, Dec. 2010.

[8] Ephraim Feigand Heidi Peterson IBM Research Yorktown Heights, NY, "Image Compression using spatial prediction",ICASSP, Vol. 4, pp.2339-2342, May 1995.

[9] Ee-Leng Tan1, Woon-Seng Gan1 and Meng-Tong Wong, "Fast Image Resizing in Discrete Cosine Transform Domain with Spatial Relationship between DCT Block and Its Sub-Blocks", IEEE ICALIP, Vol.1, pp. 1683-1687, July 2008 .

[10] G. A. Triantafyllidis, D. Tzovaras, D. Sampson, and M.G.Strintzis, "A hybrid algorithm for, the removal of blocking artifacts", IEEE Multimedia \& Expo. , Vol.1, pp.161-164, Nov. 2002.

[11] Sang-Jun Park and JechangJeong, Member, "Hybrid Image Up sampling Method in the Discrete Cosine Transform Domain",IEEE Transactions on Consumer Electronics, Vol.56, No. 4, pp.2615-2622, Nov. 2010.

[12] Wang Rui, Lin Jiangli, Li Deyu, "Edge Enhancement and Filtering of Medical Ultrasonic Images using a Hybrid Method", ICBBE Bioinformatics and Biomedical Engineering, Vol.1, pp.876 - 879, July 2007.

[13] I. O. Kirenko, R. Muijs, L. Shao,"New method for the reduction of artifacts in MPEG compressed video sequences", IEEE Multimedia \& Expo.,Vol.7,No.1, pp.469-472, Dec.2006.

[14] Shuanhu Wu, Hong Yan, and Zheng Tan,“An Efficient Wavelet-Based Deblocking Algorithm for Highly Compressed Images", IEEE Transactions on Circuits \& Systems for Video Technology.,Vol. 11, No.11, pp 1193-1198, Nov.2001.

[15] Ying Luo and Rabab K. Ward, Fellow, "Removing the Blocking Artifacts of Block-Based DCT Compressed Images", IEEE Transactions on ImageProcessing, Vol. 12 , No. 7, pp. 838-842, July 2003

[16] Kawaldeep Singh,Parveen Kumar, "Algorithm for Blocking Artifact Detection \& Reduction using adaptive filtering in Compressed Images", IJAEST, Vol. 5, No. 2, pp.156-162,

2010 